\section{ECA Rule Action}

Jonas Mellin $^{1,2}$ and Mikael Berndtsson ${ }^{1,2}$

${ }^{1}$ University of Skövde, The Informatics

Research Centre, Skövde, Sweden

${ }^{2}$ University of Skövde, School of Informatics,

Skövde, Sweden

\section{Definition}

An ECA rule action is typically arbitrary code invoked if the condition of a triggered rule evaluates to true.

\section{Key Points}

The ECA rule actions are executed in response to events triggering rules whose conditions evaluate to true. The action is executed as part of a transaction or as a transaction depending on the coupling mode in the system. One major problem is that several actions can be executed concurrently, and these may be in conflict. Another major problem is that executing an action may result in eventtriggering rules, that is, cascading rule triggering.

\section{Cross-References}

$\checkmark$ ECA Rules

- ECA Rule Condition 